\title{
Self-Organized Short-Term Memories
}

\author{
S.N. Coppersmith*, T.C. Jones ${ }^{\dagger}$, L.P. Kadanoff*, A. Levine ${ }^{\ddagger}$, J.P. McCarten ${ }^{\dagger}$, S.R. Nagel* \\ S.C. Venkataramani*, Xinlei $\mathrm{Wu}^{\dagger}$ \\ (February 14, 2018)
}

\begin{abstract}
We report short-term memory formation in a nonlinear dynamical system with many degrees of freedom. The system "remembers" a sequence of impulses for a transient period, but it coarsens and eventually "forgets" nearly all of them. The memory duration increases as the number of degrees of freedom in the system increases. We demonstrate the existence of these transient memories in a laboratory experiment.
\end{abstract}

PACS numbers: 03.20.+i, 71.45.Lr, 72.15.Nj

\footnotetext{
*The James Franck Institute, The University of Chicago, 5640 Ellis Avenue, Chicago, IL 60637

${ }^{\dagger}$ Department of Physics and Astronomy, Clemson University, Clemson, SC 29694-1911

${ }^{\ddagger}$ Exxon Research \& Engineering Company, Route 22 East, Annandale, NJ 08801
} 
We present a deterministic nonlinear dynamical system with many degrees of freedom which self-organizes to store memories, in that a configuration-dependent quantity "learns" preselected values. The system, a simple discretized diffusion equation, encodes multiple memories during an extended transient period, but, in the limit of long times, retains no more than two of them. This system thus displays a mechanism by which memories are forgotten as well as learned.

We demonstrate: (1) Short-term memories are exhibited by a system with two degrees of freedom, $N=2$, and become more pronounced as $N$ is increased. (2) The interval in which multiple memories are encoded typically grows as the square of the system's linear extent. (3) Many features of the dynamics, including their duration, can be understood analytically. (4) The mechanism is robust and is manifest in experiments on a sliding charge-density wave solid.

Consider a system of coupled maps:

$$
x_{j}(\tau+1)=x_{j}(\tau)+\operatorname{int}\left[k \sum_{i(n n)}\left(x_{i}(\tau)-x_{j}(\tau)\right)+\left(1-A_{\tau}\right)\right],
$$

where $i, j$ are the particle indices, the sum is over nearest neighbors, $\tau$ is the time index, and int $[z]$ is the largest integer less than or equal to $z$. These equations describe the evolution of the positions $x_{j}$ of $N$ particles in a deep periodic potential, with nearest neighbor particles connected by springs of spring constant $k \ll 1$, in the presence of force impulses $\left(1-A_{\tau}\right)$. They describe the dynamics of sliding charge-density waves (CDW's), [1] [1, and are closely related to models of a variety of dynamical systems. [4] The one-dimensional, $\tau$-independent version of this system $\left(A_{\tau}=A\right)$ has been studied previously [2,5](see also Ref. [3]). Here, we consider $A$ 's which repeatedly cycle through $M$ different values.

The self-organization that occurs as these maps evolve is manifest in the discrete curvature variables [5], $c_{j}(\tau)=k \sum_{i(n n)}\left(x_{i}(\tau)-x_{j}(\tau)\right)$, which obey:

$$
c_{j}(\tau+1)-c_{j}(\tau)=k \sum_{i(n n)}\left\{\operatorname{int}\left[c_{i}(\tau)+\left(1-A_{\tau}\right)\right]-\operatorname{int}\left[c_{j}(\tau)+\left(1-A_{\tau}\right)\right]\right\} .
$$

Figure 1 shows normalized histograms of $\operatorname{frac}(c)=c-\operatorname{int}(c)$ for a two-dimensional system with $M=5$, periodic boundary conditions, and a random initial configuration of 
$x$ 's. Memory encoding is shown by the accumulation of $c$ 's with $\operatorname{frac}(c)=\operatorname{frac}\left(A_{\tau}\right)$. For a while all $M$ memories are encoded to a similar degree; eventually all are forgotten except for two values of A. [6]. No evolution occurs after the last trace, a fixed point of the map (2).

Figure 2 shows the curvature variables $c_{j}(\tau)$ versus time $\tau$ for one-dimensional chains with one free and one fixed end:

$$
x_{0}(\tau)=0 ; \quad x_{N+1}(\tau)=x_{N}(\tau) \quad \text { for all } \tau
$$

During the evolution, each $c_{j}$ sticks at values corresponding to each $A_{\tau}$. This tendency is more pronounced for $N=10$ than for $N=2$, indicating that larger systems encode transient memories more effectively. At the fixed point, only one memory (rather than two as in the model with periodic boundary conditions) is encoded.

In CDW experiments, memory encoding is manifest as synchronization of the response to a repeated train of driving pulses so that $\mathrm{V} / \mathrm{I}(\mathrm{V}=$ voltage, $\mathrm{I}=\mathrm{CDW}$ current, which is proportional to the CDW velocity $v_{C D W}$ ) decreases just as each pulse ends. The correspondence between $V / I$ and the $c$ 's is discussed in detail in Refs. [2,3]. Heuristically, it follows because $x_{j}(\tau)$ can be thought of as the position of particle $j$ after pulse $\tau$, and the int functions in Eqs. (1) arise because after each pulse every particle falls into the nearest potential minimum. The memory values are at the discontinuities of the the int functions, which for the highly overdamped dynamics relevant to CDW's [1] means that many particles are at potential maxima at the end of a pulse. Since particles mounting the potential go slower than those descending it (again, implied by overdamped dynamics), when many particles have c's on memory values, then a preponderance of particles are at potential maxima, which in turn implies that the ratio $v_{C D W} / V$ is increasing at the end of each pulse. Single memory retention using identical pulses has been seen previously. [7, 8] Here we report multiple memory encoding. [9] Figure 3 shows the successful training of a sample using 5 different four-pulse sequences (current pulses). For this sample, we investigated 25 different four-pulse sequences and observed that the voltage response at the end of each pulse had a 
negative slope (indicating the retention of a memory) $85 \%$ and a positive slope only $5 \%$ of the time. Thus, multiple memories are observed in experiments as well as simulations.

To understand why the memories form, consider the "nailed" case of figure 2. Initially, each impulse causes every $x_{j}$ to increment by the same amount, so that only the spring near the nail stretches. As time progresses, this spring stretches more and more, until the force it exerts becomes large enough to keep the first particle from going into the next well under application of $A_{1}$, the impulse with the smaller fractional part. (At this point, the spring force is insufficient to change the action of $A_{2}$, the impulse with the larger fractional part.) The second spring then starts to stretch, which, on the next iteration, gives just enough added force to restore the first particle to its initial motion for both impulses. So now the first spring stretches on alternate applications of the impulse $A_{1}$, and the total spring force on particle 1 increments alternately by $+k$ and $-k$. Therefore, $c_{1}$ oscillates around the memory value $A_{1}$, leading to a plateau on the plot of figure 2. Eventually the second spring is stretched to the point that the second particle also hangs up at the impulse $A_{1}$. This, in turn, starts the stretching of the third spring, etc. A memory for $A_{1}$ is created whenever the local curvature just cancels the fractional part of $A_{1}$. A similar analysis holds for all the other impulses $A_{\tau}$ that are applied. As time progresses, the $c$ 's get stuck at all the different possible memory values.

Another way to understand why the curvatures stick at the values of $A_{\tau}$ is to note that when $c_{j}$ passes through a memory value, then the right hand side of Eq. (2) changes discontinuously, and in particular can change sign if the neighboring c's have the appropriate values. If this happens, then $c_{j}$ oscillates with amplitude $\propto k$, and sticks at the memory value. [10] This sticking can take the form of either a fixed point or a cycle in the local $c$-values.

For $k \rightarrow 0$, the dynamics separates into three regimes. The smallest motions are the $O(k)$ back-and-forth motions at the memory values, which lead to minute serrations in the plateaus that are not visible on the scale of our figures. The largest motions, involving 
changes in $c_{j}$ which are larger than of order unity, and changes in $\tau$ which are much larger than $1 / k$, are described by a linear discrete diffusion equation

$$
\tilde{c}_{j}(\tau+1)-\tilde{c}_{j}(\tau)=k \sum_{i(n n)}\left(\tilde{c}_{i}(\tau)-\tilde{c}_{j}(\tau)\right),
$$

obtained by linearizing the int functions in Eq. (22). Figure 4, which shows snapshots of configurations at two different times, demonstrates the accuracy of the linearized equation in reproducing the evolution on large scales. Numerically, the maximum deviation between the solutions to Eqs. (22) and (四) for identical initial conditions, $\sup _{j, \tau}\left|c_{j}(\tau)-\tilde{c}_{j}(\tau)\right|$, is less than unity for all system sizes, parameter values, boundary conditions, and initial conditions investigated. Using the nature of the nonlinearity in Eq. (2) together with the fact that $|z-\operatorname{int}(z)| \leq 1$ for all $z$, one can obtain a rigorous analytic bound on this difference that grows as $\log (L)$, where $L$ is the system's linear extent, [1] which is sufficient to insure the applicability of the memory duration estimates given below.

Of course, neither of the regions just discussed produces the memory effects. The memories come from an intermediate region involving variations in $c_{j}$ much larger than $k$ but smaller than unity. Figure 2 shows that, on this intermediate scale, all the $c_{j}$ 's show a very simple behavior. They are either (1) stuck at one of the memory values, or (2) are between memory values, with the $c_{j}$ varying linearly in time. This sequence of step-wise linear motions progressively reduces the variation in the discrete curvature of $c_{j}$. Thus, the intermediate and large scale motions of $c_{j}$ can be described as different kinds of diffusive smoothing.

To characterize how the memory durations depend on system parameters, first consider the case when the number of memories $M=1$. The onset time $\tau_{\text {onset }}$ is determined by the condition that the curvature of the curvature $\nabla^{2} c$ is $\sim 1$. This condition follows because the $c$ 's stick only when the discontinuity in the integer function is large enough to cause the right hand side (rhs) of Eq. (2) to change sign. For multiple memories, we sum the rhs of Eq. (2) over the $M$ terms in the cycle and note that the discontinuity occurs in only one of $M$ terms in the sum, leading to the condition $\nabla^{2} c \sim 1 / M$. For any $M$, the transient 
memories disappear when the range of curvatures becomes too small, $\delta c \equiv c_{\max }-c_{\min } \sim 1$. To relate the onset and forgetting times to these conditions on the curvatures, we use the linearized map Eq. (四), whose evolution obeys (when $\tau \gg 1 / k$ ):

$$
c_{j}(\tau)=\sum_{q} e^{i \vec{q} \cdot \vec{j}} c(q, \tau=0) e^{-k q^{2} \tau}
$$

where $c(q, \tau=0)$ is the spatial Fourier transform of the initial condition $c_{j}(\tau=0)$. For the random initial conditions for $c$ shown in figure $3, \nabla^{2} c$ is dominated by $q \sim \sqrt{k \tau}$ and the onset time $\tau_{\text {onset }} \sim c_{0} M / k$, where $c_{0}$ is a typical value of $c(q, \tau=0)$, independent of the linear extent L. At long times $\delta c$ is dominated by the longest wavelength mode, so that $\tau_{\text {forget }} \sim \frac{L^{2}}{4 \pi^{2} k} \ln \left(c_{0}\right)$. Thus, larger systems remember longer.

The system of Eqs. (2) is deterministically driven towards a fixed point. Once this point is reached, it is impossible to retrieve the short-term memories. However, it is possible to keep the transient memories from decaying by adding noise to the system. [12] For example, in the "nailed" case of Figure 2, moving the nail slowly but randomly with time creates the possibility of continuously encoding new memories. That noise can lead to the retention of memories is important for understanding our experiments. Permanently encoded multiple memories are observed, but only in samples of $\mathrm{NbSe}_{3}$ with additional conducting strips attached to the crystal between the contacts, an arrangement known to induce noise. [13]

Our memory mechanism can be compared to the "Hopfield memory," [14 which is a dynamical system with parameters adjusted so that particular configurations, which encode the desired patterns, minimize an energy functional. There, the memory is encoded in the long-time dynamics, and there is no intrinsic "forgetting" mechanism. Moreover, changing the remembered value requires nontrivial adjustment of the microscopic couplings of the model. In the CDW system studied here, the information is encoded in an evolving system, the control parameter (pulse size) is easily varied in the laboratory, and the self-organization is exhibited via standard transport measurements.

One avenue for further investigation is to characterize better the effects of noise, which, as discussed above, plays an important role in the CDW experiments. We would also like 
to identify other experimental systems which exhibit this short-term memory. Because the present model is just a discretized diffusion equation, having properties which seem quite robust, we are optimistic that physical embodiments exist that could be made into useful devices.

We thank S.E. Brown, P.B. Littlewood, M.L. Povinelli, and R. Thorne for fruitful discussions. This work was supported in part by the MRSEC program of the National Science Foundation under award number DMR-9400379. A.L. acknowledges support by an AT\&T Graduate Fellowship. 


\section{REFERENCES}

[1] See, e.g., G. Grüner, Rev. Mod. Phys. 60, 1129 (1988), R.E. Thorne, Physics Today 49, 42 (1996), and references therein.

[2] S.N. Coppersmith and P.B. Littlewood, Phys. Rev. B 36, 311 (1987).

[3] S.N. Coppersmith, Phys. Rev. A 36, 3375 (1987).

[4] P. Bak, C. Tang, and K. Wiesenfeld, Phys. Rev. Lett. 59, 381 (1987), J. Carlson and J. Langer, Phys. Rev. Lett. 62, 2632 (1989), A.V.M. Herz and J. J. Hopfield, Phys. Rev. Lett. 75, 1222 (1995).

[5] C. Tang et al., Phys. Rev. Lett. 58, 1161 (1987).

[6] Which memories are retained at long times can be determined by using the observation that at the fixed point the $c_{j}$ 's do not change under application of any $A_{\tau}$, so that (from Eqs. (1) and (2)) $P^{\prime}+\operatorname{frac}\left(A_{\tau}\right)<c_{j}<P^{\prime}+1+\operatorname{frac}\left(A_{\tau}\right)$ for some fixed integer $P^{\prime}$. The left and right inequalities are most restrictive for those $A_{\tau}$ with the largest and smallest values of $\operatorname{frac}\left(A_{\tau}\right)$, respectively.

[7] R.M. Fleming and L.F. Schneemeyer, Phys. Rev. B 33, 2930 (1986).

[8] S.E. Brown, G. Grüner, and L. Mihaly, Solid State Comm. 57, 165 (1986).

[9] Multiple memory encoding has been observed in two out of three samples of $\mathrm{NbSe}_{3}$ which have additional conducting strips attached to the crystal between the contacts. The role of these extra strips is discussed below.

[10] Ref. [5] notes the importance of the oscillations induced by the nonlinearity in the context of the fixed point selection when a single memory is encoded.

[11] S.N. Coppersmith, L.P. Kadanoff and S.C. Venkataramani, unpublished.

[12] M. Povinelli, S.N. Coppersmith, and S.R. Nagel, unpublished.

[13] M.P. Maher et al., Synthetic Metals 43, 4031 (1991).

[14] See, e.g., J.J. Hopfield, Proc. Nat. Acad. Sci. USA 79, 2554 (1982), J.J. Hopfield, Proc. Nat. Acad. Sci. USA 81, 3088 (1984), D.J. Amit, H. Gutfreund, and H. Sompolinsky, Phys. Rev. A 32, 1007 (1985), D.J. Amit, H. Gutfreund, and H. Sompolinsky, Ann. 
Phys. (NY) 173, 30 (1987). 


\section{FIGURES}

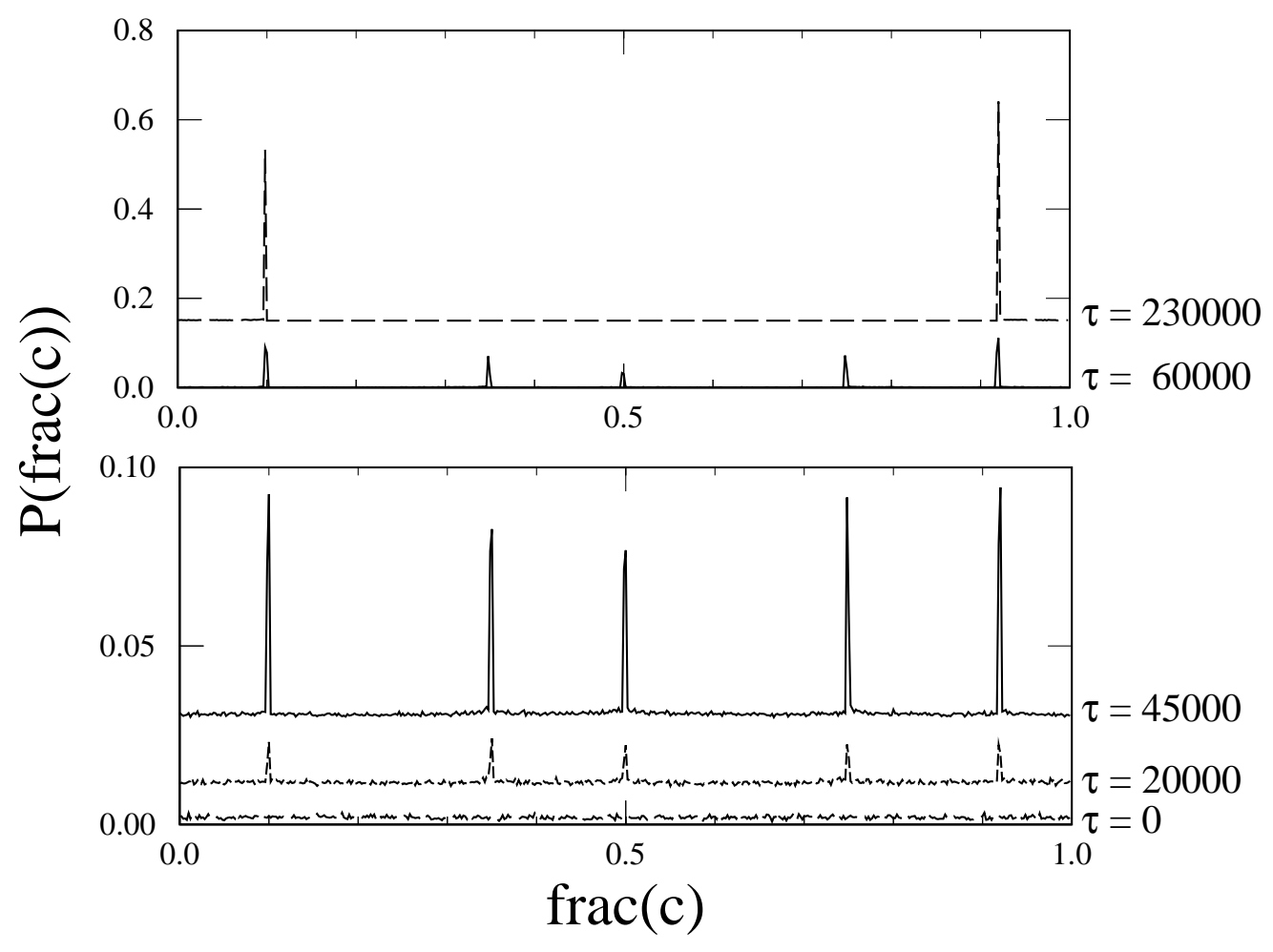

Figure 1: Memory formation of Eq. (11) in a two-dimensional $100 \times 100$ system with periodic boundary conditions, $k=0.0001$, initial condition of $x$ 's chosen randomly from the interval $[0,1000000]$, and $M=5(A=[0.1,0.35,0.5,0.75,0.92]) . P$ is the proportion of $c$ 's with fractional parts frac(c) within a bin of width 0.002. For clarity, successive curves are offset vertically. The lower panel illustrates the short-term accumulation of $c$ 's at each value of $A$; the upper panel demonstrates that at long times only two peaks persist. 


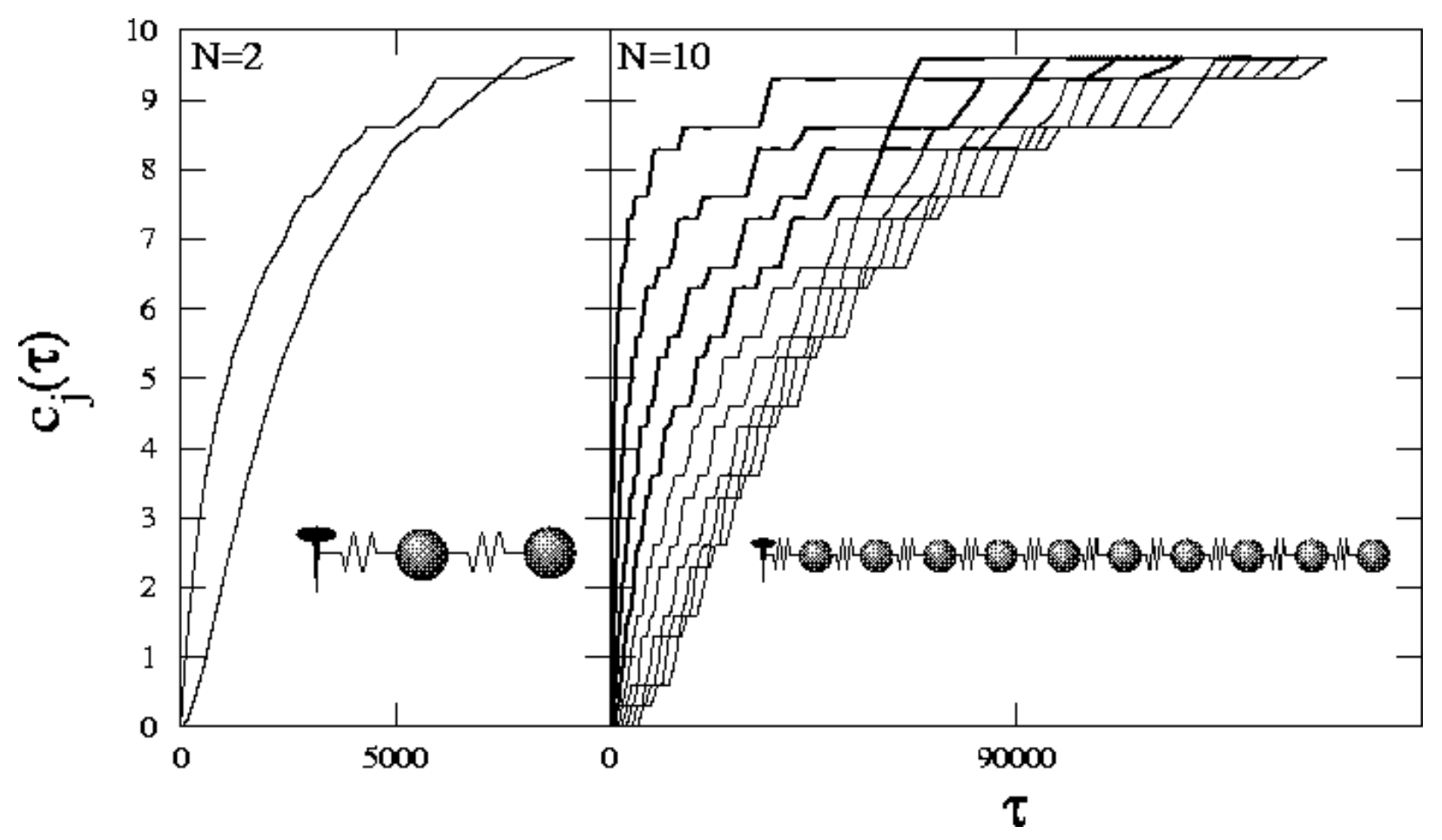

Figure 2: Plot of curvatures $c_{j}(\tau)$ versus $\tau$ for $N$-particle chains with fixed-end boundary conditions Eq. (3),$k=0.001$ and $A=[9.3,9.6]$, and initial condition $x_{j}(\tau=0)=0$ for all $j$. The memories are manifest in the plateaus (more pronounced for $N=10$ than for $N=2$ ) when the $c_{j}$ have values with fractional part of 0.3 and 0.6 . Only one memory is retained at long times. 

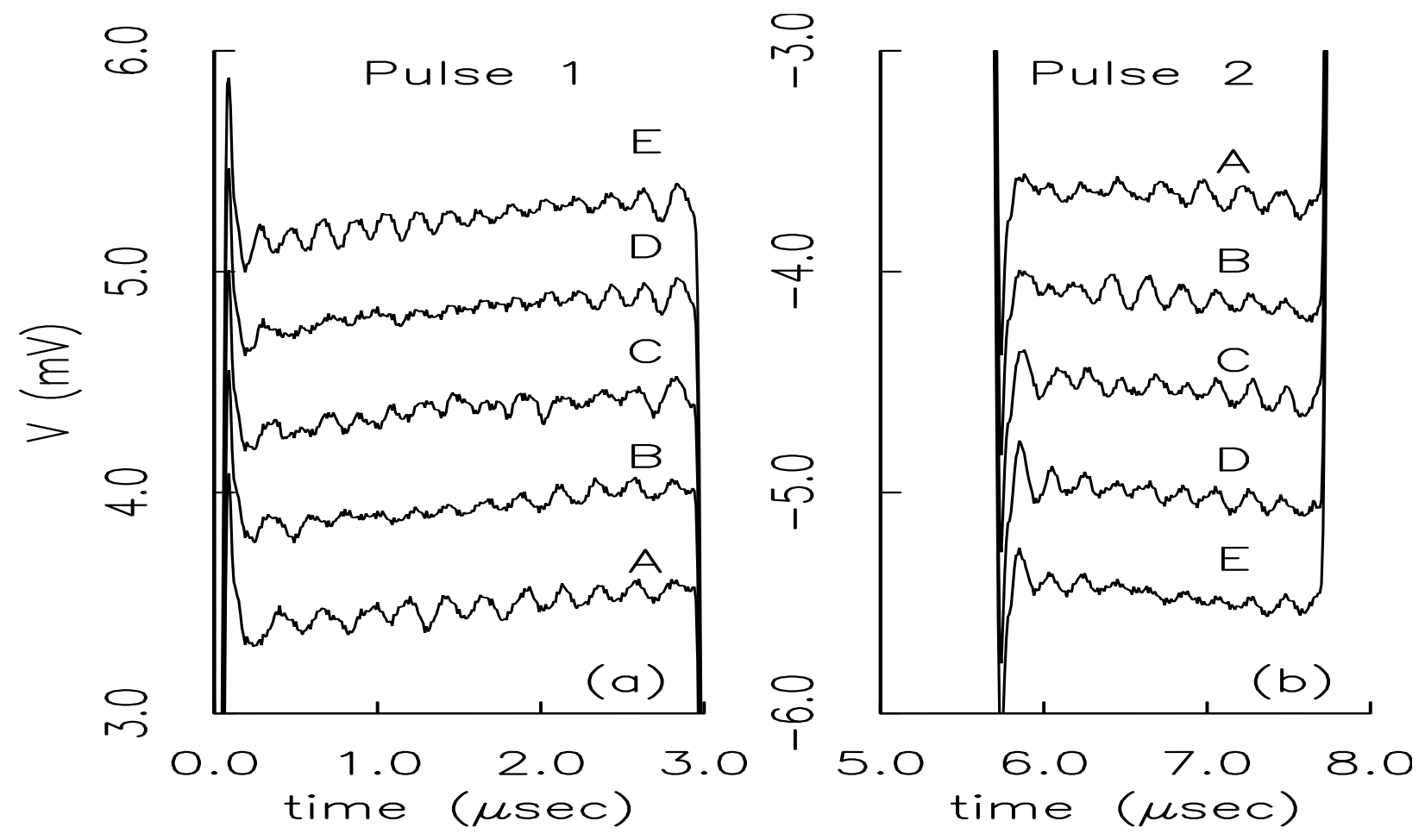

Figure 3: Trained voltage response curves of $\mathrm{NbSe}_{3}$ for five different four-pulse sequences. The evidence for multiple-memory encoding is the decreasing magnitude of the voltage at the end of each pulse. (Note the two pulses have opposite signs. The voltage response for pulses 3 and 4 are not shown.) The drive is a sequence of 4 current pulses with magnitudes $[I,-I, I,-I]$ and durations $2.95,2.05,1.75$, and $2.75 \mu s$. The drive magnitude, $I$, is 20.76, 21.16, 21.55, 21.94, and 22.33 $\mu$ A for curves A, B, C, D, and E, respectively. Training consisted of over one million pulse repetitions. Measurements were performed using a two-wire, silver-paint contact configuration. Sample dimensions are $5.2 \mu \mathrm{m}^{2} \times 980 \mu \mathrm{m}$. Additional silver paint strips of $43 \mu \mathrm{m}$ and $100 \mu \mathrm{m}$ in width are attached to the sample, centered at distances $13 \%$ and $58 \%$ between the probe contacts respectively. The curves are offset for clarity, and averaged 200 times to reduce noise. $T=50 \mathrm{~K}$, and $E_{T}=47 \mathrm{mV} / \mathrm{cm}$. 


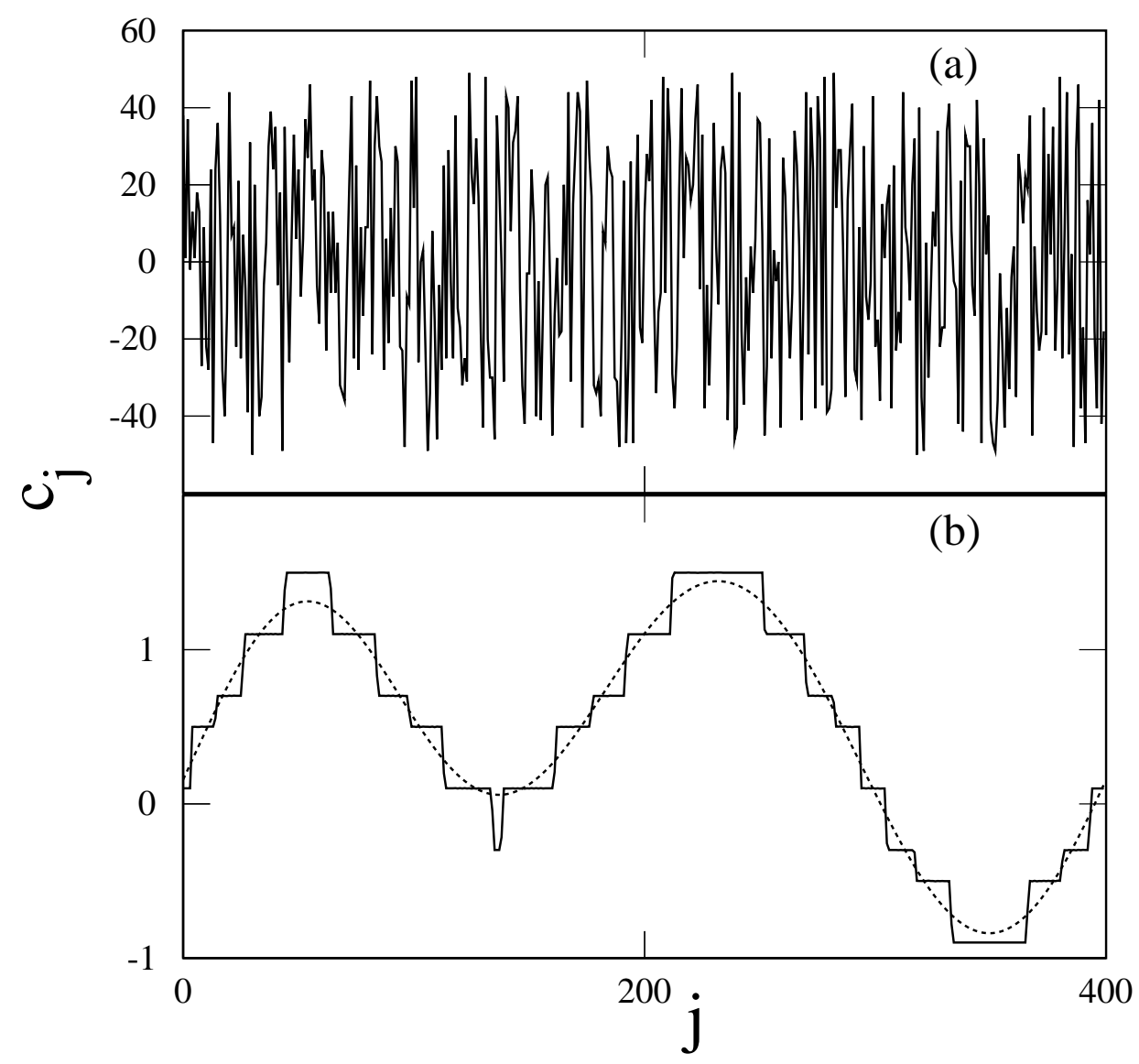

Figure 4: Snapshots of curvature $c_{j}$ versus position $j$ of a one-dimensional chain of length $\mathrm{L}=400$ with periodic boundary conditions at (a) $\tau=0$ and (b) $\tau=1200000$. (The fixed point is reached at $\tau \sim 9300000$.) Parameter values are $A=[1.1,1.5,1.7], k=0.001$. The dashed line in (b) is the configuration obtained using Eq. (田) with the same initial conditions, demonstrating that this linear diffusion equation captures accurately the large scale evolution of the system. 